\title{
ac Hopping Conduction at Extreme Disorder Takes Place on the Percolating Cluster
}

\author{
Thomas B. Schrøder and Jeppe C. Dyre \\ DNRF Centre "Glass and Time," IMFUFA, Department of Sciences, Roskilde University, Postbox 260, DK-4000 Roskilde, Denmark
}

(Received 4 January 2008; revised manuscript received 10 April 2008; published 8 July 2008)

\begin{abstract}
Simulations of the random barrier model show that ac currents at extreme disorder are carried almost entirely by the percolating cluster slightly above threshold; thus contributions from isolated lowactivation-energy clusters are negligible. The effective medium approximation in conjunction with the Alexander-Orbach conjecture lead to an excellent analytical fit to the universal ac conductivity with no nontrivial fitting parameters.
\end{abstract}

DOI: 10.1103/PhysRevLett.101.025901

PACS numbers: 66.30.Dn, 05.40.Fb, 72.80.Ng

Recent advances relating to ion conduction in glasses and other disordered solids include the application of multidimensional NMR techniques [1], the introduction of ac nonlinear spectroscopy [2], and elucidations of the high-frequency nearly constant loss [3]. Moreover, it was found that the old idea of ions moving by the vacancy mechanism may well be correct [4], and simulations gave new insight into the mixed-alkali effect [5]. Despite these significant advances, important questions remain unanswered. For instance, it is still not understood what role is played by ion interactions for the conductivity [6], or why the random barrier model (RBM) [7,8] represents ac conductivity data so well. The latter question is not answered below, but new simulations and arguments are presented that we believe lead to a fairly complete understanding of the physics of the RBM in the extreme disorder limit (low-temperature limit).

ac conductivity is studied also for amorphous semiconductors, electronically or ionically conducting polymers, defective crystals of various kinds, disordered polaronic conductors, etc. [7,8]. It is a longstanding observation that all disordered solids have remarkably similar ac conductivities [9]. Universal features include [8]: At low frequencies the conductivity is constant. At higher frequencies it follows an approximate power law with an exponent less than one that increases slightly with increasing frequency. When measured in a fixed frequency range, the exponent converges to one as temperature goes to zero. The ac conductivity is less temperature dependent than the dc conductivity and obeys time-temperature superposition (sometimes referred to as "scaling"). The frequency marking onset of ac conduction, $\omega_{m}$, has the same activation energy as the dc conductivity.

These and other observed features are reproduced by the RBM that is characterized $[8,10]$ by five assumptions: (i) All charge carrier interactions including self-exclusion are ignored; (ii) Charge carrier motion takes place on a cubic lattice; (iii) All lattice sites have same energy; (iv) Only nearest-neighbor jumps are allowed; (v) Jump rates $\propto \exp \left(-E / k_{B} T\right)$ have random activation energies with distribution $p(E)$. In the RBM the ac conductivity $\sigma(\omega)$ relative to $\sigma(0)$ as a function of a suitably scaled frequency becomes independent of $p(E)$ in the extreme disorder limit, i.e., when the width of $p(E)$ is much larger than $k_{B} T$ [8]. Despite having no nontrivial free parameters the RBM universal ac conductivity gives a good fit to experiment [8]; more refined models yield results that are close to those of the RBM [11].

It is well known that the percolation threshold determines the dc conductivity activation energy [12]. At low temperatures the particles preferably jump across the lowest barriers. The highest barriers on the percolation cluster are bottlenecks dominating the low-temperature dc conductivity. If $E_{c}$ is the highest barrier on the percolating cluster, one has $\sigma(0) \sim \exp \left(-E_{c} / k_{B} T\right)$ as $T \rightarrow 0$ [12]. In order to have a nonzero dc conductivity of the percolation cluster, barriers slightly above the percolation threshold must be included. This defines the "fat percolation cluster" [8]; on length scales shorter than its correlation length the fat percolation cluster appears fractal, on longer length scales it appears homogeneous.

Understanding the RBM universal ac conductivity in terms of percolation arguments is much more challenging. Traditionally $[7,13]$ the problem was approached "from the high-frequency side" by proceeding as follows. For frequencies $\omega>\omega_{m}$ there is a characteristic activation energy $E(\omega)<E_{c}$ for motion on time scales $\sim 1 / \omega$; when $\omega$ decreases towards $\omega_{m}$ one has $E(\omega) \rightarrow E_{c}$. Links with $E \leq E(\omega)$ form finite low-activation-energy clusters. The cluster size distribution is assumed to determine the ac conductivity. Some time ago we proposed what amounts to coming "from the low-frequency side", namely, that all relevant motion takes place on a subset of the infinite fat percolation cluster [8]. Numerical evidence for this conjecture is given below, where it is shown that contributions from low-activation energy clusters outside the fat percolating cluster are insignificant. Moreover, it is shown that by assuming that not just a subset, but in fact the entire fat percolation cluster contributes significantly, an excellent analytical approximation to the universal ac conductivity with no nontrivial fitting parameters may be derived.

The simulations of the RBM reported below refer to the Box distribution of activation energies $\left(p(E)=1 / E_{0}\right.$ for 
$0<E<E_{0}$, zero otherwise); ac universality in the extreme disorder limit implies that this distribution gives representative results [8]. The lowest temperature simulated is given by $\beta=320$ where $\beta$ is the inverse dimensionless temperature, $\beta \equiv E_{0} / k_{B} T$. For $\beta=320$ the jump rates cover almost 140 orders of magnitude, making simulations quite challenging. We used a method based on solving the Laplace transform of the master equation numerically [14]. Conductivity data for $\beta=320$ give an excellent representation of the universal master curve for the RBM over the frequency range studied here [8].

In previously reported simulations [8] we applied an activation energy cut-off above the percolation threshold, $E_{\text {cut }} / E_{0}=p_{c}+k / \beta$, where $p_{c}=0.2488$ is the percolation threshold for the cubic lattice and $k$ a numerical constant. Jump rates for links with activation energies larger than $E_{\text {cut }}$ were set to zero in order to be able to simulate large samples. Figure 1(a) presents the real part of the ac conductivity $\sigma(\omega)=\sigma^{\prime}(\omega)+i \sigma^{\prime \prime}(\omega)$ for $k=3.2$ and $k=12.8$, respectively, at $\beta=320$. There is little difference between the two data sets. The dashed line gives the prediction of the effective medium approximation combined with the Alexander-Orbach conjecture as detailed below [Eq. (2)]. Figure 1(b) gives the relative errors involved for different $k$ values, taking $k=12.8$ as repre-

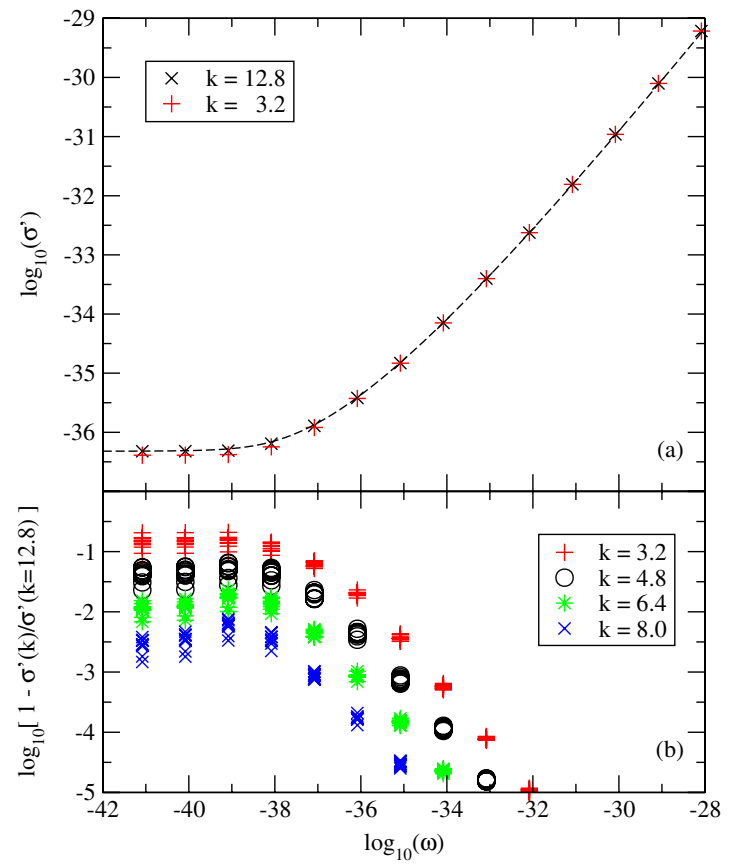

FIG. 1 (color online). Results for the ac conductivity at $\beta \equiv$ $E_{0} / k_{B} T=320$ in rationalized units [8]; in these units the frequency marking onset of ac conduction, $\omega_{m}$, is of order $10^{-38}$. Ten independent $96 \times 96 \times 96$ samples were simulated. (a) Real part of $\sigma(\omega)$ with two cutoff's: $k=3.2$ and $k=12.8$, averaged over the ten samples. The dashed line is the prediction of Eq. (2) scaled to the $k=12.8$ data. (b) Relative deviation from $k=12.8$ as a function of frequency plotted for each of the ten independent samples. senting the "correct" data. The errors are largest in the dc regime and decrease with increasing $k$. Choosing $k=6.4$ gives an error of just $1 \%-2 \%$.

We proceed to investigate the behavior with $k=6.4$ in more detail. Applying this cutoff, the links with nonzero jump rate fall into two sets, the fat percolating cluster and all remaining finite isolated clusters. The latter do not contribute to the dc conductivity. According to the traditional approaches based on cluster statistics, however, they give a significant contribution to the ac conductivity as soon as $\omega \gtrsim \omega_{m}[7,13]$. This was never tested numerically, though. Figure 2 presents the contribution from isolated clusters $\sigma_{\text {IC }}^{\prime}(\omega)$ relative to the full ac conductivity as a function of the real part of the scaled conductivity $\tilde{\sigma} \equiv$ $\sigma(\omega) / \sigma(0)$. The dashed lines mark this relative mass of the isolated clusters for $\beta=160$ and $\beta=320$, respectively. The quantity $\sigma_{\text {IC }}^{\prime}(\omega) / \sigma^{\prime}(\omega)$, however, is much smaller than the relative mass of isolated clusters for the range of frequencies covered in the figure, i.e., up to $10^{10}$ times $\omega_{m}$ (compare Fig. 1). For $\beta \rightarrow \infty$ the relative mass of isolated clusters goes to one, whereas we find that $\sigma_{\mathrm{IC}}^{\prime}(\omega) / \sigma^{\prime}(\omega)$ is independent of temperature and stays insignificant. In summary, the dominant part of the low-temperature universal ac conductivity comes from the fat percolation cluster [8] with little contribution from isolated clusters.

We now turn to the issue of analytical approximations utilizing the effective medium approximation (EMA) $[10,15,16]$. If $G \equiv \int_{0}^{\infty} P_{0}(t) \exp (-i \omega t) d t$, where $P_{0}(t)$ is the probability for a particle to be at a site given it was there at $t=0$ for a homogeneous system with uniform jump rate, the extreme disorder limit of the EMA self-

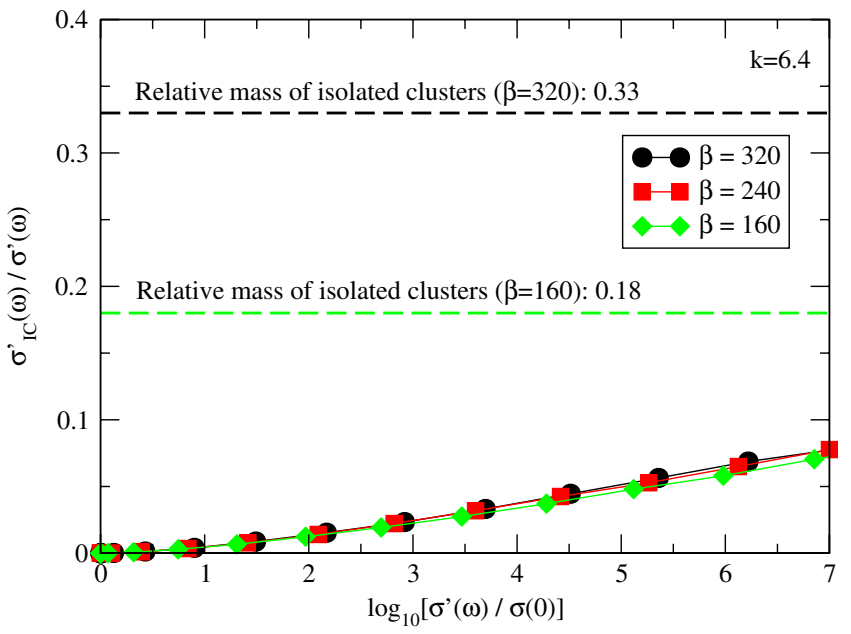

FIG. 2 (color online). Contribution from isolated clusters for the real part of the ac conductivity, $\sigma_{\mathrm{IC}}^{\prime}(\omega)$, relative to $\sigma^{\prime}(\omega)$ as a function of the scaled real part of the conductivity, $\tilde{\sigma}^{\prime}(\omega) \equiv$ $\sigma^{\prime}(\omega) / \sigma(0)$ (cutoff: $k=6.4$ ). The two dashed lines mark the relative masses of isolated clusters, i.e., low-activation-energy links outside the fat percolation cluster. Their contribution to the conductivity is much smaller than their relative mass, showing that the dominant part of the ac conduction takes place on the fat percolation cluster. 
consistency equation is $\ln \tilde{\sigma}=\Lambda \beta i \omega G$, where $\Lambda$ is a numerical constant [10]. This determines a frequencydependent complex "effective" jump rate that is proportional to the frequency-dependent conductivity $[7,10]$. Henceforth we switch to the rationalized unit system [8] where the EMA self-consistent ac conductivity equals the complex effective jump rate. Because $P_{0}(t)$ is a function of the effective jump rate times time, $\sigma t$, the quantity $i \omega G$ is a function of $i \omega / \sigma$. In the frequency range relevant for the universal ac conductivity of the extreme disorder limit corresponding to times obeying $\sigma t \gg 1$, one has $|i \omega G| \ll$ $1[10,17]$. If $d$ is dimension, whenever $d \geq 2 i \omega G$ as a function of $i \omega / \sigma$ has a regular first order term [7,10]: $i \omega G=\alpha_{1}(i \omega / \sigma)+\ldots$ If $\Lambda$ is absorbed into a dimensionless frequency by defining $\tilde{\omega} \equiv \alpha_{1} \Lambda \beta \omega / \sigma(0)$, the EMA universality equation $[10,17]$ for $d \geq 2$ is

$$
\tilde{\sigma} \ln \tilde{\sigma}=i \tilde{\omega} .
$$

This equation gives a qualitatively correct, but numerically inaccurate fit to simulations [8].

In our previous works [8] it was proposed that some unspecified subset of the fat percolating cluster with fractal dimension $d_{f}$ ("the diffusion cluster") is responsible for the ac conduction. If $d_{f}<2$ this led to the diffusion cluster approximation (DCA): $\ln \tilde{\sigma}=(i \tilde{\omega} / \tilde{\sigma})^{d_{f} / 2}$ [8]. If the diffusion cluster is the so-called backbone, one expects $d_{f}=$ 1.7, if the diffusion cluster is the set of red bonds, one expects $d_{f}=1.1$ [8]. Treating $d_{f}$ as a fitting parameter led to $d_{f}=1.35$ [8], leaving the nature of the diffusion cluster as an open problem.

What if not just a subset, however, but the entire fat percolating cluster contributes significantly to the universal ac conductivity? Random walks on a fractal structure are characterized by $P_{0}(t) \propto(\sigma t)^{-d_{H} / 2}$ [18] where $d_{H}$ is the spectral dimension. For $d_{H}<2$ this leads to $i \omega G \propto$ $(i \omega / \sigma)^{d_{H} / 2}$. In terms of a suitably scaled frequency the EMA thus implies the DCA expression with $d_{f}=d_{H}$. According to the Alexander-Orbach conjecture [19]known to be almost correct (see, e.g., [14,20]) —one has $d_{H}=4 / 3$ for the infinite percolating cluster. If the frequency is suitably scaled, this leads to the following approximation to the universal ac conductivity of the extreme disorder limit:

$$
\ln \tilde{\sigma}=\left(\frac{i \tilde{\omega}}{\tilde{\sigma}}\right)^{2 / 3} .
$$

As shown in Fig. 1(a) this expression provides an excellent fit to the universal ac conductivity of the extreme disorder limit [21]. Equation (2) may be put to a more stringent test, however, than just fitting the real part of $\tilde{\sigma}(\tilde{\omega})$. Figure 3(a) tests one implication of Eq. (2), $|\ln \tilde{\sigma}|=|\tilde{\omega} / \tilde{\sigma}|^{2 / 3}$, by plotting $|\ln \tilde{\sigma}|$ as a function of $|\tilde{\omega} / \tilde{\sigma}|$ in a log-log plot. A crossover between two powerlaw regimes is seen, corresponding to a crossover between Eqs. (1) and (2). In Fig. 3(b) the apparent exponent
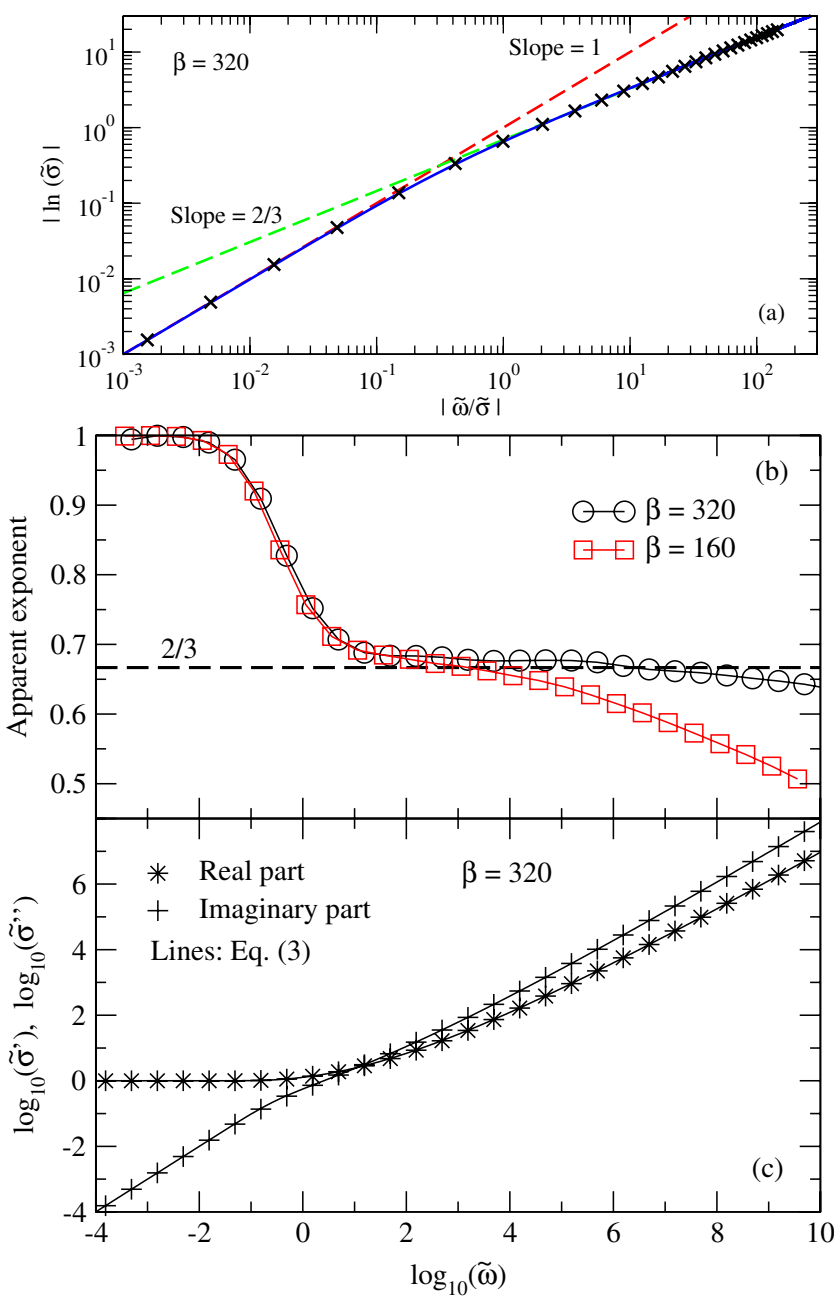

FIG. 3 (color online). Testing Eqs. (2) and (3). Data represent averaging over 100 independent $96 \times 96 \times 96$ samples $(\beta=$ 320, cutoff: $k=6.4$ ) and 100 independent $64 \times 64 \times 64$ samples $(\beta=160$, cutoff: $k=6.4)$. For both temperatures the frequency was empirically scaled such that $\tilde{\sigma}=1+\tilde{\omega}$ in the low-frequency limit, where $\tilde{\sigma} \equiv \sigma(\omega) / \sigma(0), \tilde{\omega} \equiv \omega \Delta \epsilon \epsilon_{0} / \sigma(0)$ [8]. (a) $|\ln \tilde{\sigma}|$ as a function of $|\tilde{\omega} / \tilde{\sigma}|$ in a $\log$-log plot. (b) The apparent exponent $d \ln (|\ln \tilde{\sigma}|) / d \ln |\tilde{\omega} / \tilde{\sigma}|$ as a function of scaled frequency. A crossover from fractal behavior (exponent $2 / 3$ ) to homogeneous behavior (exponent one) is clearly visible. (c) The real and imaginary parts of the scaled conductivity compared to Eq. (3) (full lines).

$d \ln (|\ln \tilde{\sigma}|) / d \ln |\tilde{\omega} / \tilde{\sigma}|$ is plotted as a function of scaled frequency. Similar results are found by plotting the ratio of the phases of the complex numbers $\ln \tilde{\sigma}$ and $i \tilde{\omega} / \tilde{\sigma}$ (data not shown).

The picture emerging from Figs. 3(a) and 3(b) is the following: Equation (2) works well whenever $\tilde{\omega} \gtrsim 1$; here the fat percolation cluster appears fractal because over one cycle the particles move less than the correlation length. At low frequencies there is a transition to the analytic behavior predicted when the dimension is larger than 2 [Eq. (1)]; over one cycle the particles here move longer than the correlation length and, consequently, the fat percolation cluster appears homogeneous. 
The entire frequency range is accurately described by the expression

$$
\ln \tilde{\sigma}=\frac{i \tilde{\omega}}{\tilde{\sigma}}\left(1+2.66 \frac{i \tilde{\omega}}{\tilde{\sigma}}\right)^{-1 / 3}
$$

that is plotted as the full lines in Figs. 3(a) and 3(c). The exponent $-1 / 3$ was chosen to get agreement with Eq. (2) for $|\tilde{\omega} / \tilde{\sigma}| \gg 1$. The difference between Eqs. (2) and (3) is significant only at such low frequencies that $\tilde{\sigma}(\tilde{\omega})$ is of order unity [Fig. 3(a)]. Equation (2) breaks down for the imaginary part for $\tilde{\omega}<1$ where Eq. (2) predicts $\tilde{\sigma}^{\prime \prime} \propto \tilde{\omega}^{2 / 3}$ instead of the observed $\tilde{\sigma}^{\prime \prime} \propto \tilde{\omega}$. Numerical solutions of Eqs. (2) and (3) are provided in Ref. [22].

In our opinion, the RBM may now be regarded as solved in the extreme disorder limit in the sense that a good understanding of the model's physics is at hand, leading to an accurate description of the universal ac conductivity. A notable consequence of the above is that the EMAgenerally believed to be inaccurate except at weak disorder-works surprisingly well in the extreme disorder limit if the "geometrical" input $G$ is taken to reflect the fractal geometry of the percolation cluster. It would be interesting to know whether similar results are found when the EMA is applied for the extreme disorder limit of other models.

The centre for viscous liquid dynamics "Glass and Time" is sponsored by the Danish National Research Foundation (DNRF).

[1] M. Vogel, C. Brinkmann, H. Eckert, and A. Heuer, Phys. Rev. B 69, 094302 (2004); S. Berndt, K. R. Jeffrey, R. Kuchler, and R. Böhmer, Solid State Nucl. Magn. Reson. 27, 122 (2005); R. Böhmer, K. R. Jeffrey, and M. Vogel, Prog. Nucl. Magn. Reson. Spectrosc. 50, 87 (2007).

[2] B. Roling, J. Chem. Phys. 117, 1320 (2002); A. Heuer, S. Murugavel, and B. Roling, Phys. Rev. B 72, 174304 (2005).

[3] C. Leon, A. Rivera, A. Varez, J. Sanz, J. Santamaria, and K. L. Ngai, Phys. Rev. Lett. 86, 1279 (2001); B. Roling, C. Martiny, and S. Murugavel, ibid. 87, 085901 (2001); D. L. Sidebottom and C. M. Murray-Krezan, ibid. 89, 195901 (2002).

[4] A. N. Cormack, J. Du, and T.R. Zeitler, Phys. Chem. Chem. Phys. 4, 3193 (2002); H. Lammert, M. Kunow, and A. Heuer, Phys. Rev. Lett. 90, 215901 (2003); J. C. Dyre, J. Non-Cryst. Solids 324, 192 (2003).

[5] H. Lammert and A. Heuer, Phys. Rev. B 72, 214202 (2005).

[6] W. Dieterich and P. Maass, Chem. Phys. 284, 439 (2002); M. Schulz, W. Dieterich, and P. Maass, Z. Phys. Chem. (Muenchen) 218, 1375 (2004).

[7] J. M. Stevels, in Handbuch der Physik, edited by S. Flügge (Springer, Berlin, 1957), Vol. 20, p. 350; H.E. Taylor, J. Soc. Glass Technol. 41, 350T (1957); B. I. Shklovskii and A.L. Efros, Electronic Properties of Doped Semiconductors (Springer, Berlin, 1984); H. Böttger and V.V. Bryksin, Hopping Conduction in Solids (Akademie, Berlin, 1985); J. W. Haus and K. W. Kehr, Phys. Rep. 150,
263 (1987); J.-P. Bouchaud and A. Georges, ibid. 195, 127 (1990); M.P. J. van Staveren, H.B. Brom, and L. J. de Jongh, ibid. 208, 1 (1991); Charge Transport in Disordered Solids, edited by S. Baranovski (Wiley, New York, 2006).

[8] J.C. Dyre and T.B. Schrøder, Rev. Mod. Phys. 72, 873 (2000); T. B. Schrøder and J. C. Dyre, Phys. Rev. Lett. 84, 310 (2000); J. C. Dyre and T. B. Schrøder, Phys. Status Solidi B 230, 5 (2002); T. B. Schrøder and J. C. Dyre, Phys. Chem. Chem. Phys. 4, 3173 (2002).

[9] J. O. Isard, J. Non-Cryst. Solids 4, 357 (1970); H. Namikawa, ibid. 18, 173 (1975); A. K. Jonscher, Nature (London) 267, 673 (1977); A. E. Owen, J. Non-Cryst. Solids 25, 370 (1977); A. Mansingh, Bull. Mater. Sci. 2, 325 (1980); S. Summerfield, Philos. Mag. B 52, 9 (1985).

[10] J. C. Dyre, J. Appl. Phys. 64, 2456 (1988); J.C. Dyre, Phys. Rev. B 49, 11709 (1994).

[11] M. Porto, P. Maass, M. Meyer, A. Bunde, and W. Dieterich, Phys. Rev. B 61, 6057 (2000); O. Bleibaum, H. Böttger, and V. V. Bryksin, J. Phys. Condens. Matter 15, 1719 (2003); M. Sahimi, M. Naderian, and F. Ebrahimi, Phys. Rev. B 71, 094208 (2005); W.F. Pasveer, P. A. Bobbert, and M. A. J. Michels, ibid. 74, 165209 (2006); E. Pazhoohesh, H. Hamzehpour, and M. Sahimi, ibid. 73, 174206 (2006); T. Ishii and E. Matsumoto, J. Phys. Soc. Jpn. 76, 024606 (2007).

[12] V. Ambegaokar, B. I. Halperin, and J. S. Langer, Phys. Rev. B 4, 2612 (1971); B. I. Shklovskii and A. L. Efros, Zh. Eksp. Teor. Fiz. 60, 867 (1971) [Sov. Phys. JETP 33, 468 (1971)]; S. Tyc and B. I. Halperin, Phys. Rev. B 39, 877 (1989).

[13] H. Böttger, V. V. Bryksin, and G. Y. Yashin, J. Phys. C 12, 3951 (1979); I. P. Zvyagin, Phys. Status Solidi B 97, 143 (1980); S. D. Baranovskii and H. Cordes, J. Chem. Phys. 111, 7546 (1999); A. G. Hunt, Philos. Mag. B 81, 875 (2001).

[14] T. B. Schrøder, Europhys. Lett. 81, 30002 (2008).

[15] D. A. G. Brüggeman, Ann. Phys. (Leipzig) 416, 636 (1935).

[16] In Ref. [10] we wrote $\tilde{G}$ for what is here denoted $G$. This reserves the use of tildes to dimensionless quantities.

[17] V. V. Bryksin, Fiz. Tverd. Tela (Leningrad) 22, 2441 (1980) [Sov. Phys. Solid State 22, 1421 (1980)].

[18] D. Ben-Avraham and S. Havlin, Diffusion and Reactions in Fractals and Disordered Systems (Cambridge University Press, Cambridge, England, 2000).

[19] S. Alexander and R. Orbach, J. Phys. (Paris), Lett. 43, L625 (1982).

[20] B. D. Hughes, Random Walks and Random Environments (Clarendon, Oxford, 1996).

[21] Equation (2) implies that the real part of the ac conductivity a few decades above $\omega_{m}$ has an approximate powerlaw frequency depedence with exponents in the range $0.6-$ 0.7 , thus close to $2 / 3$ (compare Fig. 1) and consistent with experiment. It is important to note that this fact is not related to the exponent $2 / 3$ appearing in Eq. (2); for instance Eq. (1) has the same mathematical property.

[22] See EPAPS Document No. E-PRLTAO-101-030829 for two files which provide numerical solutions to Eqs. (2) and (3), respectively. For more information on EPAPS, see http://www.aip.org/pubservs/epaps.html. 